\section{Successful conversion of fibroblasts into liver cells}

Japanese researchers have demonstrated that fibroblasts can be successfully converted into hepatocyte-like (iHep) cells that mature to fully functional hepatocytes in vivo, and that a combination of only two transcription factors-Hnf4a plus Foxa1, Foxa2 or Foxa3-are needed for this conversion to take place.

Hepatocytes account for $\sim 70 \%$ of the liver. Numerous liver-enriched transcription factors are essential for the induction of hepatocytes from hepatic progenitor cells during liver development, but existing evidence suggests that hepatocytes can also emerge from a number of different cell types and tissues in response to particular stimuli or after transplantation into the liver. In their study published in Nature, Sayaka Sekiya and Atsushi Suzuki questioned what these crucial hepatocyte-specific factors were and sought to identify the factors required to directly induce fibroblasts into hepatocytes.

The researchers forced the expression of hepatic-fate-inducing factors in mouse fibroblasts using retroviruses before culturing the transduced cells under specific conditions to mediate hepatocyte differentiation. They found that when $\mathrm{Hnf} 4 a$ was expressed alongside either Foxa1, Foxa2 or Foxa3, fibroblasts were reprogrammed into iHep cells that proliferated in vitro and possessed specific morphological and functional features of hepatocytes, including the presence of glycogen stores as well as the expression of genes encoding liver enzymes and sets of genes involved in fat, cholesterol and glucose metabolism, among others. Moreover, in a mouse model of liver failure, iHep cells were shown to reconstitute damaged liver tissue after transplantation. Mutant mice treated with fibroblasts died within 27 days after transplantation whilst $40 \%$ of those that received iHep cell transplants survived for $>10$ weeks (similar length of survival to hepatocyte-transplanted mice).

Whether iHep cells can be generated from human fibroblasts remains to be seen, but the findings from this study

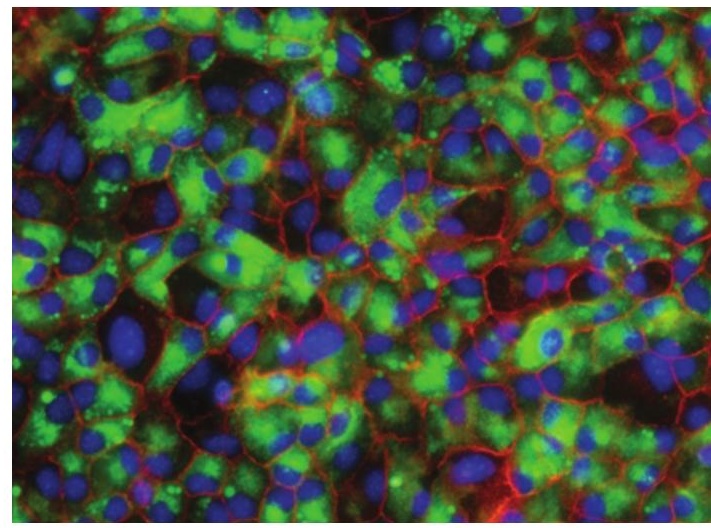

iHep cells derived from adult mouse skin fibroblasts. Courtesy of A. Suzuki.

could not only improve understanding of hepatocyte differentiation, but also pave the way for new therapies for liver disease. "Human iHep cells could be used for the development of therapeutic strategies for hepatocyte transplantation, artificialorgan hepatic support and screening of the pharmacological effects of drugs," says Suzuki.

\section{Katrina Ray}

Original article Sekiya, S. \& Suzuki, S. Direct conversion of mouse fibroblasts to hepatocyte-like cells by defined factors. Nature 475, 390-393 (2011) 